\title{
A GENERAL BOUND FOR THE NET PREMIUM OF THE LARGEST CLAIMS REINSURANCE COVERS
}

\author{
ERHARD KREMER
}

Universität Hamburg

\begin{abstract}
For a general class of reinsurance treaties the author gives an upper bound for the net premium. This result can be seen as the counterpart to a premium bound for the classical stop-loss reinsurance cover (see Bowers, 1969). For some special cases some preliminary work can be found in Kremer (1983).
\end{abstract}

\section{INTRODUCTION}

Over the past six years the author has investigated the problem of calculating the premium for general reinsurance treaties defined with the order statistic of the observed claims. The starting point was from papers of AMMETER (1964), BENKTANDER (1978), BERLINER (1972) and KUPPER (1971), treating two special cases under very special model assumptions, and a more general paper of CIMINELLI (1976). In 1984 the author developed general formulas for the net premium of the generalized reinsurance treaties under asymptotic considerations, in 1985 corresponding non-asymptotic formulas (see KREMER 1984, 1985) and a handy recursive rating method in 1986 (see KREMER, 1986b). Special cases were given by the author in 1982 and 1986 (see KREMER, 1982, 1986a). For generalized claim number distributions some results can be found in KREMER (1988). Some years ago, in 1983, the author gave asymptotic upper bounds for the net premium of two special types of reinsurance covers. From these results the question arises as to whether it is possible to give upper premium bounds also for the generalized type of reinsurance treaty defined with ordered claims. In the following these bounds are derived under fairly general conditions.

\section{THE GENERAL REINSURANCE TREATY}

Let the random variables $X_{1}, X_{2}, \ldots$ denote the claims of a collective of risks and let $N$ describe the claim number. We order the claims in decreasing size resulting in the ordered claims

$$
X_{N: 1} \geqslant X_{N: 2} \geqslant \ldots \geqslant X_{N: N} .
$$

For given constants $c_{1}, c_{2}, \ldots$ such that

$$
\sum_{i=1}^{n} c_{i} y_{i} \in\left[0, \sum_{i=1}^{n} y_{i}\right]
$$

ASTIN BULLETIN Vol. 18, No. 1 
holds true for all

$$
y_{1} \geqslant y_{2} \geqslant \cdots \geqslant y_{n} \geqslant 0,
$$

we define the claim amount taken by the reinsurer by

$$
R_{N}=\sum_{i=1}^{N} c_{i} X_{N: i}
$$

Consequently the family $\left(c_{i}, i \geqslant 1\right)$ defines a reinsurance treaty, which we call a generalized largest claims reinsurance cover (formerly: reinsurance treaty based on ordered claims). Two well-known special cases are given in the following examples.

EXAMPLE 1. We take

$$
\begin{aligned}
& c_{1}=c_{2}=\cdots=c_{p}=1 \\
& c_{j}=0, \quad \forall j>p
\end{aligned}
$$

and get the classical largest claims treaty with parameter $p$, which covers the $p$ largest claims of a collective.

EXAMPLE 2. Let us choose

$$
\begin{aligned}
& c_{1}=c_{2}=\cdots=c_{p-1}=1 \\
& c_{p}=1-p \quad \text { and } \quad c_{j}=0, \quad \forall j>p,
\end{aligned}
$$

defining the so-called ECOMOR-treaty with parameter $p$, covering the $p$ largest claims in excess of the $p$ th largest claim.

The reader is invited to give more obscure examples (see KREMER, 1984).

\section{THE GENERAL PREMIUM BOUND}

We look at the net premium $N R P$ of our general treaty defined by the family $\left(c_{i}, i \geqslant 1\right)$, i.e. the expected value of $R_{N}$,

and get the main result.

$$
N R P=E\left(R_{N}\right)
$$

THEOREM 1. With the above notation we assume that the claim sizes are identically distributed, mutually independent and independent of the claim number $N$. We get the upper bound for the net premium

$$
N R P \leqslant \mathrm{E}\left(N \bar{c}_{N}\right) \mu+\sqrt{E\left(N(N-1) s_{N}^{2}\right)} \sigma
$$

with

$$
\begin{gathered}
\mu=E\left(X_{i}\right), \sigma^{2}=\operatorname{Var}\left(X_{i}\right) \\
\bar{c}_{N}=\left(\frac{1}{N}\right) \sum_{i=1}^{N} c_{i}, s_{N}^{2}=\left(\frac{1}{N-1}\right) \sum_{i=1}^{N}\left(c_{i}-\bar{c}_{N}\right)^{2},
\end{gathered}
$$

and assuming the existence of the moments in (3.1). 
ProOF. With the Cauchy-Schwarz inequality we get

$$
\sum_{i=1}^{N}\left(c_{i}-\bar{c}_{N}\right)\left(X_{N: i}-\mu\right) \leqslant\left(\sum_{i=1}^{N}\left(c_{i}-\bar{c}_{N}\right)^{2} \sum_{i=1}^{N}\left(X_{N: i}-\mu\right)^{2}\right)^{1 / 2} .
$$

We take the conditional expectation, leading with Jensen's inequality to

$$
E\left(\sum_{i=1}^{N}\left(c_{i}-\bar{c}_{N}\right)\left(X_{N: i}-\mu\right) \mid N\right) \leqslant\left[\sum_{i=1}^{N}\left(c_{i}-\bar{c}_{N}\right)^{2} E\left(\sum_{i=1}^{N}\left(X_{i}-\mu\right)^{2} \mid N\right)\right]^{1 / 2} .
$$

The left-hand side is equal to

$$
E\left(\sum_{i=1}^{N} c_{i}\left(X_{N: i}-\mu\right) \mid N\right)
$$

and the right-hand side equal to

$$
\left(\sum_{i=1}^{N}\left(c_{i}-\bar{c}_{N}\right)^{2} N\right)^{1 / 2} \sigma
$$

Now taking the unconditional expectation, applying once again Jensen's inequality and rearranging leads to the statement.

REMARK 1. For nonrandom $N=n$ the statement of Theorem 1 corresponds with a result in DAVID (1980), p. 78-79.

REMARK 2. Obviously the above result can be generalized to treaties where in (2.1) $X_{N: i}$ is replaced by $h\left(X_{N: i}\right)$ with a measurable function $h$ on the claims $X_{N: i}$. In the Theorem 1 one only has to replace $X_{i}$ by $h\left(X_{i}\right)$ in the formulas for $\mu$ and $\sigma^{2}$.

REMARK 3. Notice that the above premium bound depends only on the first and second moment of the claim size distribution and on the distribution of the claim number $N$. For the practical application first one has to estimate the mean $\mu$ and the variance $\sigma^{2}$ by the classical mean and variance estimators based on the past claims experience. Since

$$
\begin{gathered}
E\left(N \bar{c}_{N}\right)=\sum_{n=0}^{\infty}\left(\operatorname{Prob}(N=n) \sum_{i=1}^{n} c_{i}\right), \\
E\left(N(N-1) s_{N}^{2}\right)=\sum_{n=0}^{\infty} n\left(\operatorname{Prob}(N=n) \sum_{i=1}^{n}\left(c_{i}-\bar{c}_{n}\right)^{2}\right),
\end{gathered}
$$

one also needs estimators for $\operatorname{Prob}(N=n)$ with $n \geqslant 1$. This can be done by using the empirical distribution function of the known past claim numbers or alternatively by applying well-known parametric statistical estimation methods based for example on the assumption of Poisson-distributed claim numbers (see LEHMANN, 1983).

As an illustration we cite the following examples. 
EXAMPLE 3. We take the conditions of the Example 1, this means that we investigate the classical largest claims cover. Then one has

$$
N \bar{c}_{N}= \begin{cases}p, & \text { if } N>p \\ N, & \text { if } N \leqslant p\end{cases}
$$

and

$$
N(N-1) s_{N}^{2}= \begin{cases}p(N-p), & \text { if } N>p \\ 0, & \text { if } N \leqslant p .\end{cases}
$$

Consequently we have

$N R P \leqslant p \mu \operatorname{Prob}(N>p)+E(N \mid N \leqslant p) \mu \operatorname{Prob}(N \leqslant p)$

$$
+((E(N \mid N>p)-p) p \operatorname{Prob}(N>p))^{1 / 2} \sigma,
$$

and if $\operatorname{Prob}(N \leqslant p) \approx 0$ we have the approximation

$$
N R P \leqq p \mu+\sqrt{ }(E(N)-p) p \sigma .
$$

EXAMPLE 4. We take the ECOMOR-treaty of Example 2 of Section 2. We get

$$
N \bar{c}_{N}= \begin{cases}0, & \text { if } N \geqslant p \\ N, & \text { if } N<p\end{cases}
$$

and

$$
N(N-1) s_{N}^{2}= \begin{cases}N p(p-1), & \text { if } N \geqslant p \\ 0, & \text { if } N<p .\end{cases}
$$

This implies the inequality

$$
\begin{aligned}
N R P \leqslant E(N \mid N<p) \operatorname{Prob}(N & <p) \mu \\
& +\left((E(N \mid N \geqslant p) \operatorname{Prob}(N \geqslant p) p(p-1))^{1 / 2} \sigma,\right.
\end{aligned}
$$

and in the case that

$$
\operatorname{Prob}(N<p) \approx 0,
$$

we get the approximate bound

$$
N R P \leqq \sqrt{E(N) p(p-1) \sigma .}
$$

These formulas are correct for each finite collective, whereas the formulas in KREMER $(1982,1983,1984)$ are derived for growing collectives with asymptotic considerations.

\section{AN ASYMPTOTIC PREMIUM RATE BOUND}

We assume that we have a sequence of growing collectives such that the claim 
number $N_{k}$ of the $k$ th collective satisfies

$$
\left\{\begin{array}{l}
\lim _{k \rightarrow \infty}\left[E\left(N_{k}\right)\right]=+\infty \\
\lim _{k \rightarrow \infty}\left(\frac{\overline{\operatorname{Var}\left(N_{k}\right)}}{E\left(N_{k}\right)}\right)=0
\end{array}\right.
$$

and that the claims $X_{i}, i \geqslant 1$ in each collective are mutually independent, independent of $N_{k}$ and indentically distributed with expected value $\mu$ and variance $\sigma^{2}$. The generalized largest claims treaty is defined in the collective no. $k$ by the sequence of constants

$$
\left(c_{i}^{(k)}, i \geqslant 1\right)
$$

dependent on $k$ and the claim amount (2.1) with $c_{i}^{(k)}$ instead of $c_{i}$. For $n=1,2,3, \ldots$ and a sequence $\left(c_{i}^{(k)}, i \geqslant 1\right)$ we define a step-function $c_{k n}$ on $(0,1]$ by:

$$
c_{k n}(u)=c_{i}^{(k)}, \quad \text { for } u \in\left(\frac{i-1}{n}, \frac{i}{n}\right] \text { and } i=1,2,3, \ldots, n \text {. }
$$

We assume that the family of functions $\left(c_{k n}, k \geqslant 1, n \geqslant 1\right)$ is uniformly bounded and that there exists a bounded function $c$ on $(0,1]$ such that for each sequence $\left(n_{k}, k \geqslant 1\right)$ with $n_{k} \in\{1,2, \ldots\}$ and

$$
\left\{\begin{array}{l}
\lim _{k \rightarrow \infty}\left(n_{k}\right)=\infty \\
\lim _{k \rightarrow \infty}\left(\frac{n_{k}}{E\left(N_{k}\right)}\right)=1
\end{array}\right.
$$

one has (with the Lebesque-integral) the convergence

$$
\lim _{k \rightarrow \infty}\left(\int_{0}^{1}\left(c_{k / n_{k}}(u)-c(u)\right)^{2} d u\right)=0 .
$$

We denote with $U_{k}$ the upper premium bound

$$
U_{k}=E\left(N_{k} \bar{c}_{N_{h}}\right) \mu+\sqrt{E\left(N_{k}\left(N_{k}-1\right) S_{N_{k}}^{2}\right)} \sigma
$$

for the $k$ th collective in Theorem 1 . The mean total claim amount in the $k$ th collective is defined by

$$
\nu_{k}=E\left(N_{k}\right) \mu .
$$

With these notations we get the following elegant result:

THEOREM 2. Under the above conditions we have the convergence

$$
\lim _{k \rightarrow \infty}\left(\frac{U_{k}}{\nu_{k}}\right)=\mu(c)+\left(\frac{\sigma}{\mu}\right) \sqrt{s(c)}
$$


with

$$
\begin{aligned}
& \mu(c)=\int_{0}^{1} G(u) d u \\
& s(c)=\int_{0}^{1}(G(u)-\mu(c))^{2} d u .
\end{aligned}
$$

Proof. One has the equality

$$
\left(\frac{U_{k}}{\nu_{k}}\right)=E\left(\frac{N_{k}}{E\left(N_{k}\right)} \bar{c}_{N_{k}}\right)+\left(\frac{\sigma}{\mu}\right) \sqrt{E\left(\frac{N_{k}\left(N_{k}-1\right)}{E\left(N_{k}\right)^{2}} s_{N_{k}}^{2}\right)} .
$$

Without loss of generality we have because of (4.1)

$$
\left\{\begin{array}{l}
\lim _{k \rightarrow \infty}\left(N_{k}\right)=+\infty \quad \text { a.e. } \\
\lim _{k \rightarrow \infty}\left(\frac{N_{k}}{E\left(N_{k}\right)}\right)=+1 \quad \text { a.e. }
\end{array}\right.
$$

and because of this and (4.2), (4.3)

$$
\begin{cases}\lim _{k \rightarrow \infty}\left(\bar{c}_{N_{k}}\right)=\mu(c) & \text { a.e. } \\ \lim _{k \rightarrow \infty}\left(s_{N_{k}}^{2}\right)=s(c) & \text { a.e. }\end{cases}
$$

implying with a version of the theorem of dominated convergence (see LOEVE, 1963 , p. 162) the result of the theorem above.

REMARK 4. Notice that (4.1) and (4.2), (4.3) are nothing else but more illustrative sufficient conditions for (4.5) and (4.6).

REMARK 5. Similar arguments and asymptotic premium formulas are given in a very general paper of the author (see KREMER, 1984), generalizing some results in KREMER (1982).

As an illustration we cite the following case.

EXAMPLE 5. We take the classical largest claims cover of the Example 1 with parameter $p_{k}$ in the $k$ th collective, assume $(4.1)$ and that with a given $s \in(0,1)$ the following holds:

$$
\lim _{k \rightarrow \infty}\left(\frac{p_{k}}{E\left(N_{k}\right)}\right)=s
$$

This implies (4.3) under the condition (4.2) with

$$
c(u)= \begin{cases}1, & \text { for } u \leqslant s \\ 0, & \text { for } u>s\end{cases}
$$


which means

$$
\begin{aligned}
& \mu(c)=s \\
& s(c)=s(1-s)
\end{aligned}
$$

and consequently the Theorem 2 yields

$$
\lim _{k \rightarrow \infty}\left(\frac{U_{k}}{\nu_{k}}\right)=s+\sqrt{s(1-s)}\left(\frac{\sigma}{\mu}\right)
$$

or alternatively

$$
\lim _{k \rightarrow \infty}\left(\frac{U_{k}}{\nu_{K}}\right)=\lim _{k \rightarrow \infty}\left[\left(\frac{p_{k}}{E\left(N_{k}\right)}\right)+\sqrt{\left(\frac{p_{k}}{E\left(N_{k}\right)}\right)\left(1-\left(\frac{p_{k}}{E\left(N_{k}\right)}\right)\right)}\left(\frac{\sigma}{\mu}\right)\right] .
$$

This means that the premium rate

$$
\rho=\left(\frac{N R P}{E(N) \mu}\right)
$$

of the largest claims treaty covering the $p$ largest claims in a collective with expected claim number $E(N)$, expected value $\mu$ and variance $\sigma^{2}$ of the claim size can asymptotically be bounded by the expression

$$
\left(\frac{p}{E(N)}\right)+\sqrt{\left(\frac{p}{E(N)}\right)\left(1-\left(\frac{p}{E(N)}\right)\right)}\left(\frac{\sigma}{\mu}\right) .
$$

\subsection{A numerical Example}

We assume Pareto-distributed claim sizes, i.e.

$$
P\left(X_{i} \leqslant x\right)=1-x^{-\alpha}, \quad \text { for } x \geqslant 1,
$$

with a parameter $\alpha>2$, implying

$$
(\sigma / \mu)=(\alpha(\alpha-2))^{-1 / 2} .
$$

We get for the numerical values of the premium rate bound (4.8) (in per cent) the following results:

(a) for $\alpha=2.5$

\begin{tabular}{r|rrrrrrr} 
& $p=1$ & 2 & 3 & 4 & 5 & 6 & 7 \\
\hline$E(N)=100$ & 9.90 & 14.52 & 18.26 & 21.53 & 24.49 & 27.24 & 29.82 \\
200 & 6.81 & 9.90 & 12.37 & 14.52 & 16.46 & 18.26 & 19.94 \\
400 & 4.72 & 6.81 & 8.47 & 9.90 & 11.19 & 12.37 & 13.48 \\
800 & 3.28 & 4.72 & 5.84 & 6.81 & 7.67 & 8.47 & 9.20 \\
1600 & 2.30 & 3.28 & 4.05 & 4.72 & 5.30 & 5.84 & 6.34
\end{tabular}


KREMER

(b) for $\alpha=3.0$

\begin{tabular}{r|rrrrrrr} 
& $p=1$ & 2 & 3 & 4 & 5 & 6 & 7 \\
\hline$E(N)=100$ & 6.74 & 10.08 & 12.84 & 15.31 & 17.58 & 19.71 & 21.73 \\
200 & 4.57 & 6.74 & 8.52 & 10.08 & 11.51 & 12.84 & 14.11 \\
400 & 3.13 & 4.57 & 5.73 & 6.74 & 7.66 & 8.52 & 9.32 \\
800 & 2.16 & 3.13 & 3.90 & 4.57 & 5.17 & 5.73 & 6.25 \\
1600 & 1.51 & 2.16 & 2.68 & 3.13 & 3.53 & 3.90 & 4.24
\end{tabular}

(c) for $\alpha=3.5$

\begin{tabular}{r|rrrrrrr} 
& $p=1$ & 2 & 3 & 4 & 5 & 6 & 7 \\
\hline$E(N)=100$ & 5.34 & 8.11 & 10.44 & 12.55 & 14.51 & 16.36 & 18.13 \\
200 & 3.58 & 5.94 & 6.80 & 8.11 & 9.31 & 10.44 & 11.52 \\
400 & 2.43 & 3.58 & 4.51 & 5.34 & 6.10 & 6.80 & 7.47 \\
800 & 1.66 & 2.42 & 3.04 & 3.58 & 4.06 & 4.51 & 4.94 \\
1600 & 1.15 & 1.67 & 2.08 & 2.43 & 2.75 & 3.04 & 3.32
\end{tabular}

Some years ago the author published a very special bound for the net premium rate of the largest claims cover (see KREMER, 1983), i.e.

$$
\left(\frac{p}{E(N)}\right)+\left(\frac{1}{E(N)}\right) \sum_{j=1}^{p}\left((j-1-2) / 2^{2 j-1}\right)^{1 / 2}\left(\frac{\sigma}{\mu}\right) .
$$

The corresponding numerical values are tabulated in table 1 of the former publication. One notices that the bound (4.8) is better than the bound (4.9) for larger $p$ values, whereas it becomes worse for smaller $p$ values. Consequently it is preferable to use the minimum of the formulas (4.8) and (4.9) as an upper bound for the net premium of the largest claims reinsurance cover.

Obv:ously Theorem 2 is fairly general. Nevertheless there are examples where some of the conditions are not satisfied. We look at the following.

EXAMPLE 6. We investigate the ECOMOR-cover of Example 2 with parameter $p_{k}$ in the $k$ th collective. Unfortunately our conditions of Theorem 2 are not suitable, we have to prove (4.6) directly. Again let $N_{k}$ be the claim number in the collective no. $k$. Instead of (4.7) we assume that

$$
\lim _{k \rightarrow \infty}\left(\frac{p_{k}^{2}}{E\left(N_{k}\right)}\right)=t
$$

for a number $t \in(0, \infty)$. Since we have by the calculations in Example 4

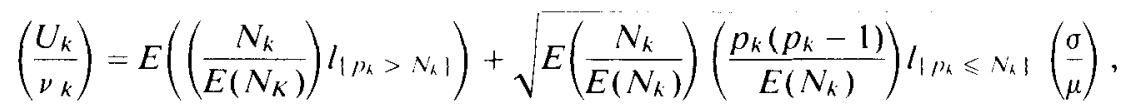

where $l \cdot$, denotes the indicator function of the set $\{\cdot\}$. As a consequence we get 
as in the proof of Theorem 2 under the conditions (4.1) (or (4.5))

$$
\lim _{k \rightarrow \infty}\left(\frac{U_{k}}{\nu_{k}}\right)=\sqrt{t}\left(\frac{\sigma}{\mu}\right)=\lim _{k \rightarrow \infty} \sqrt{\frac{p_{k}\left(p_{\mathrm{k}}-1\right)}{E\left(N_{k}\right)}}\left(\frac{\sigma}{\mu}\right) .
$$

In other words the premium rate $\rho$ (see above) of the ECOMOR-treaty, covering the $p$ largest claims in excess of the $p$ largest claim, can asymptotically be bounded by the expression:

$$
\sqrt{\left(\frac{p(p-1)}{E(N)}\right)}\left(\frac{\sigma}{\mu}\right)
$$

with the expected claims number $E(N)$, the expected value $\mu$ and the standard deviation $\sigma$ of the claim sizes. Clearly we can assume as in the numerical part of Example 5, that the claim sizes are Pareto-distributed and can give numerical results. These can be compared with those derived with formula (4.1) in KREMER (1983). This evaluation is left to the interested reader. It turns out that the bound (4.10) is uniformly higher than the asymptotic bound in KREMER (1983).

\section{ACKNOWLEDGEMENT AND COMMENTS}

The author thanks the referees for several helpful comments, improving the present paper. A first version of the paper was presented at the last Oberwolfach meeting on Risk Theory, September 1987.

\section{REFERENCES}

AMMETER, H. (1964) The rating of the "Largest claim" reinsurance covers. Quarterly Letter Jubilee Number 79-109.

BENKTANDER, G. (1978) Largest claims reinsurance (LCR). A quick method to calculate LCR-risk rates from excess of loss risk rates. ASTIN Bulletin 10, 54-58.

BERLIINER, B. (1972) Correlations between excess of loss reinsurance covers and reinsurance of the $n$ largest claims. ASTIN Bulletin 6, 260-275.

Bowers, N. L. (1969) An upper bound on the stop-loss net premium. Transactions of the Society of Actuaries 21, 211-217.

CIMINEI.I. E. (1976) On the distribution of the highest claims and its application to the automobile insurance liability. Transactions of the 20-th International Congress of Actuaries 501-517.

DAvid, H. A. (1980) Order Statistics. John Wiley, New York.

KREMER, E. (1982) Rating of the largest claims and ECOMOR reinsurance treaties for large portfolios. ASTIN Bulletin 13, 47-56.

KREMER, E. (1983) Distribution-free upper bounds on the premiums of the LCR and ECOMOR treaties. Insurance: Mathematics and Economics 2, 209-213.

KREMER, E. (1984) An asymptotic formula for the net premium of some reinsurance treaties. Scandinavian Actuarial Journal 11-22.

KREMER, E. (1985) Finite formulae for the premium of the general reinsurance treaty based on ordered claims. Insurance: Mathematics and Economics 4, 233-238.

Krimer, E. (1986a) Simple formulas for the premiums of the LCR and ECOMOR treaties under exponential claim sizes. Blätter der deutschen Gesellschaft für Versicherungsmathematik XVII, $237-243$.

KREMER, E. (1986b) Recursive calculation of the net premium for largest claims reinsurance covers. ASTIN Bulletin 16, 101-108. 
KREMER, E. (1988) Reinsurance premiums under generalized claim number distributions. To appear in the Transactions of the 23rd International Congress of Actuaries, Helsinki 1988.

KUPPER, J. (1971) Contributions to the theory of the largest claims cover. ASTIN Bulletin 6, 134- 146.

Lehmann, E. L. (1983) Theory of Point Estimation. John Wiley, New York.

LOĖve, M. (1963) Probability Theory. Van Nostrand, New York.

Prof. Dr. E. KREMER

Institut für Mathematische Stochastik, Universität Hamburg, D-2000 Hamburg 13, Germany 